\title{
Thin resonant structures for angle and polarization independent microwave absorption
}

\author{
Matthew J. Lockyear, ${ }^{\text {a) }}$ Alastair P. Hibbins, J. Roy Sambles, Peter A. Hobson, ${ }^{\text {b) }}$ and \\ Christopher R. Lawrence b) \\ School of Physics, University of Exeter, Stocker Road, Exeter EX4 4QL, United Kingdom
}

(Received 23 September 2008; accepted 3 December 2008; published online 28 January 2009)

\begin{abstract}
We present a microwave absorbing structure comprised of an array of subwavelength radius copper disks, closely spaced from a ground plane by a low loss dielectric. Experiments and accompanying modeling demonstrate that this structure supports electromagnetic standing wave resonances associated with a cylindrical cavity formed by the volume immediately beneath each metal disk. Microwave absorption on resonance of these modes, at wavelengths much greater than the thickness of the structure, is dictated almost entirely by the radius of the disk and permittivity of the dielectric, being largely independent of the incident angle and polarization. (C) 2009 American Institute of Physics. [DOI: 10.1063/1.3059568]
\end{abstract}

Research into resonant cylindrical dielectric cavity antennas (dielectric elements mounted on a metallic ground plane) was conducted in the microwave bands as early as 1983. ${ }^{1}$ Since then a large number of theoretical and experimental investigations have been undertaken, resulting in a host of applications including microantennas for mobile communications and wireless networks. ${ }^{2-4}$ Some researchers also explored resonators with a metallic "cap" that reduce the cavity's fundamental resonance frequency., ${ }^{5,6}$ While most studies focus on microstrip or aperture coupled single cavity resonators, we consider here a two-dimensional array of closely spaced cavities, supporting resonances that provide strong absorption at frequencies determined by the geometry of the unit cell.

The use of structured metal-dielectric interfaces to provide frequency selective absorption of incident radiation is certainly not new. For instance, the current authors investigated dual period bigratings ${ }^{7}$ that provide efficient incident angle independent absorption. Other examples include Dallenbach layers ${ }^{8}$ and Salisbury screens. ${ }^{9}$ However, these types of surfaces are often limited to a minimum thickness of one quarter wavelength. While Hibbins et al. ${ }^{10}$ and more recently Landy et al. ${ }^{11}$ used structured metal-dielectric composites to overcome this thickness constraint, these structures provide a polarization dependent response. Here we investigate a periodic array of cavities that support resonant modes with no quantization requirement in the direction along the axis of the cavity. This allows the construction of an ultrathin and flexible structure whose electromagnetic (EM) response is not significantly perturbed by the angle or polarization of the incident beam. Such a surface has many applications in a wide variety of commercial sectors, from radar absorbing materials and EM shielding applications to increasing the energy transfer efficiency in solar cells. ${ }^{12-14}$

The sample [Fig. 1(a), inset] consists of a 500 $\times 500 \mathrm{~mm}^{2}$ polyester sheet (permittivity of $\varepsilon=3.2+0.01 i$ and thickness of $\left.t_{s p}=100 \mu \mathrm{m}\right)$ coated on each side with a copper layer with thickness of $t_{p}=18 \mu \mathrm{m}$. Copper is then

\footnotetext{
a) Electronic mail: m.j.lockyear@exeter.ac.uk.

${ }^{b)}$ Also at QinetiQ, Cody Technology Park, Farnborough, Hampshire GU14 OLX, United Kingdom.
}

removed from one surface via standard print and etch techniques to give a square array of circular disks with radius of $a=3 \mathrm{~mm}$ whose centers are spaced in the plane by $\lambda_{g}$ $=6.2 \mathrm{~mm}$. The experimental apparatus utilizes a spherical mirror to collimate and steer a plane incident wave from a transmitting horn antenna onto the sample. The sample is

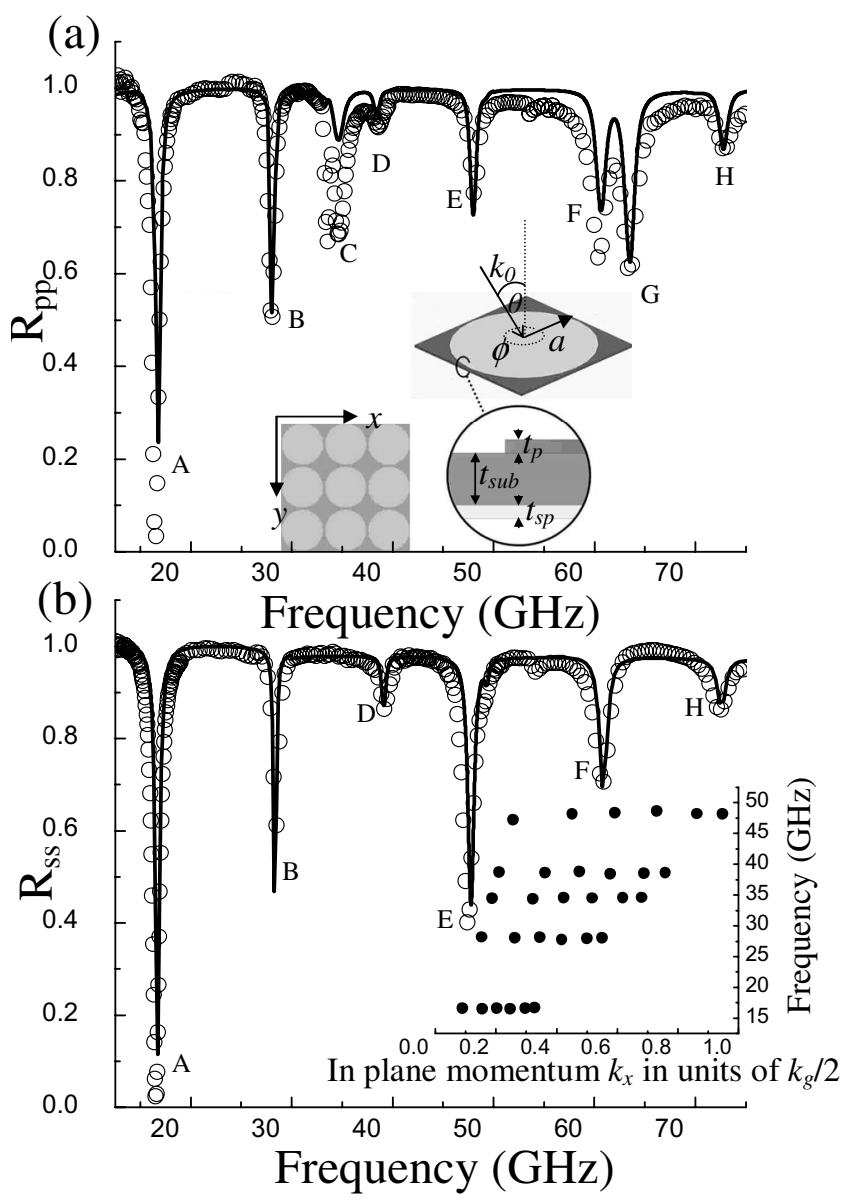

FIG. 1. Measured reflectivity as a function of frequency (circles) for (a) $p$-polarized and (b) $s$-polarized incident plane wave radiation at $\theta=26.25^{\circ}$, $\phi=0^{\circ}$. Also shown are the predictions of a finite element model (solid line). (a) Inset: a schematic of the experimental sample. (b) Inset: the resonant frequency of the first five modes supported by the sample plotted as a function of in-plane momentum $\left(k_{x}\right)$. 

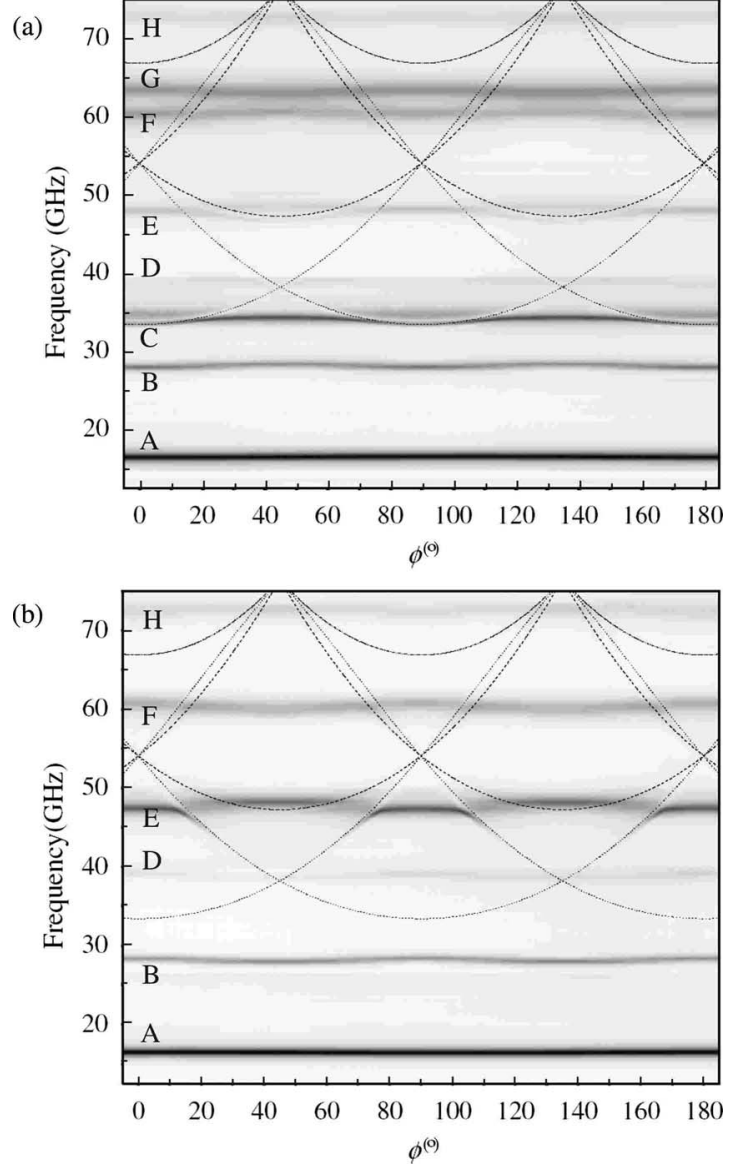

FIG. 2. Measured reflectivity as a function of azimuth angle for (a) $p$-polarized and (b) $s$-polarized incident radiation at $\theta=26.25^{\circ}$. Light areas correspond to high reflection while dark areas correspond to strong absorption. Also shown are the diffracted light lines (dotted lines) arising from $[1,0],[0,1]$, and $[1,1]$ scatterings.

mounted on a rotating turntable allowing data to be obtained over a full range of azimuthal angles $\phi$ for discrete angles of incidence $\theta$ in the range of $10^{\circ}<\theta<70^{\circ}$. The reflected signal is normalized to that of a flat copper plate of the same surface area.

Figure 1 (circles) shows specular reflectivity as a function of frequency for [Fig. 1(a)] p-polarized and [Fig. 1(b)] $s$-polarized incident plane wave radiation at $\theta=26.5^{\circ}$ and $\phi$ $=0^{\circ}$. Up to eight resonant modes are clearly visible as reflectivity minima, which are labeled $\mathrm{A}-\mathrm{H}$. Also shown are the predictions from a commercially available finite element method (FEM) model (solid line). ${ }^{15}$ We modeled the response of the structure by assuming that the complex permittivity of the substrate does not vary over the studied frequency range. Despite this rather oversimplistic assumption, the comparison between the experimental data and the predictions from the numerical model is excellent. However, the width of resonance predicted by the model is less than that observed experimentally, in part due to a spread in the experimental parameters of the sample. Note that the fundamental resonance $(A)$, is the most strongly coupled, with $95 \%$ of the incident radiation being absorbed on resonance [experimental measurements (not included) show polarization conversion to be negligible].

The dispersion of modes $A-E$ is also shown [Fig. 1(b), inset]. Here, reflectivity as a function of frequency is recorded over a range of incident angles. In-plane momentum

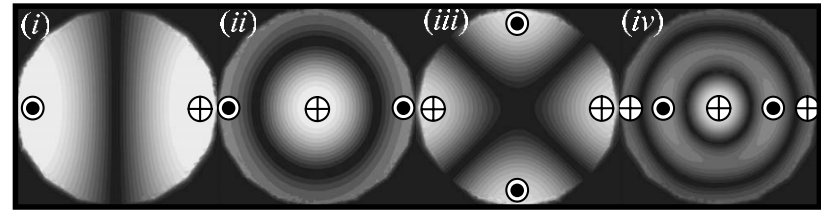

FIG. 3. (i)-(iv) show the time averaged electric field strength on resonance for modes $A, B, C$, and $G$, respectively. Field strengths are calculated at $\theta$ $=26.25^{\circ}$ and $\phi=0^{\circ}$.

$\left(k_{x}\right)$ is then extracted and plotted as a function of frequency, clearly showing that each mode is largely nondispersive with incident angle $\theta$.

Figure 2 shows the measured specular reflection from the sample for [Fig. 2(a)] p-polarization and [Fig. 2(b)] $s$-polarization as a function of both frequency and $\phi$. The diffracted light lines arising from $[1,0],[0,1]$, and $[1,1]$ scatterings are also shown. Note the azimuthal independence of the fundamental mode $(A)$. Higher order modes, while still strongly localized, exhibit some perturbation due to their interaction with the light lines. For example, notice the splitting of mode $C$ in Figs. 1(a) and 2(a). Also note that modes $C$ and $G$ are absent from the $s$-polarized response. In order to fully appreciate the nature of all the modes supported by this structure, it is useful to investigate the EM fields on resonance of the individual modes using the FEM model.

Parts (i) and (ii) of Fig. 3 show the time averaged electric field magnitudes associated with modes $A(16.1 \mathrm{GHz})$ and $B(27.2 \mathrm{GHz})$, respectively, calculated with $p$-polarized incident radiation at $\theta=26.25^{\circ}$ and $\phi=0^{\circ}$. Here, fields are evaluated over the $x y$-plane positioned midway between the copper disk and ground plane. The direction of the electric vector at a point in phase corresponding to maximum field enhancement is also indicated. First we note that each element of the array acts as a cavity resonator. In addition, each disk is circular and there is little interaction with its neighbors (increasing the pitch does not significantly perturb the frequency of the resonances). Hence one observes a frequency response that is almost entirely independent of azimuth angle and pitch.

The confinement of the fields pertaining to the fundamental mode under each patch is further demonstrated by Fig. 4. Here we see field enhancement ( $E$ and $H$, respectively) as a function of distance through the unit cell. Field magnitudes in this instance are calculated over a line parallel to the $x$-axis (the same orientation as the incident electric vector) passing through the center of the patch, midway between the patch and ground plane. It is clear that the $E$ - and $H$-fields are confined to the region under each patch (shaded region) and decay rapidly where the dielectric spacer layer is unbounded by the patch. This suggests that the localization of the mode is purely due to geometry, arising from a modeshaped mismatch between the mode under the patch and the propagating plane wave in free space. ${ }^{16}$

Further inspection of Fig. 3 [parts (i)-(iv)] shows that the resonant field solutions for the modes supported bear a striking resemblance to the eigenmodes of a long (but truncated) metallic-walled waveguide of the same cross sectional geometry. ${ }^{17}$ There is, however, one important difference: the roles of the electric and magnetic vectors are reversed, due to the EM boundary conditions of the thin disk cavity being the inverse of those of a truncated metal-clad waveguide of similar cross section. Specifically the two open ends of a tradi- 


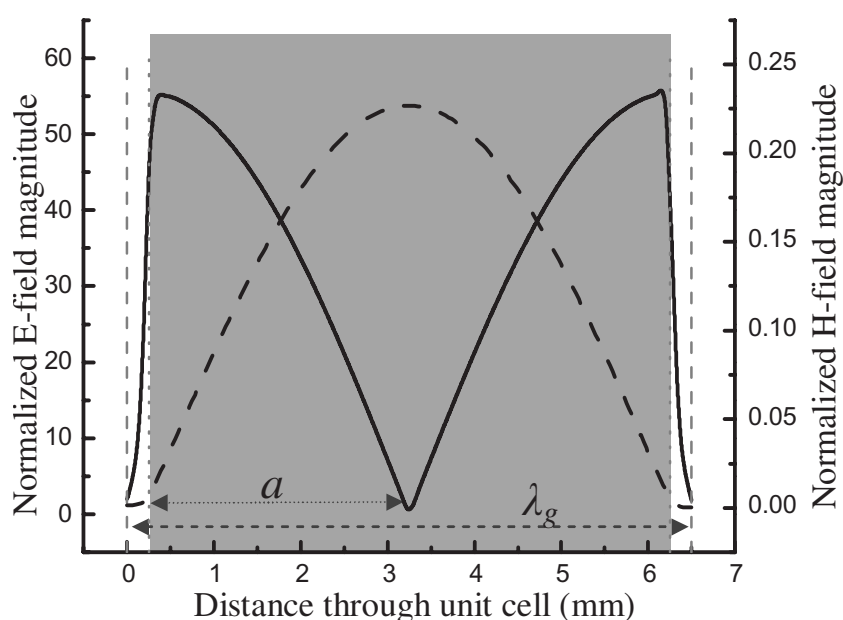

FIG. 4. (a) $E$-field and (b) $H$-field magnitude (normalized to an incident $E$-field of unity) as a function of distance through the unit cell along a line parallel to the $x$-axis passing midway between the patch and ground plane at $y=0$ (patch center). Fields are calculated at a phase corresponding to the maximum enhancement at the fundamental resonant frequency of the structure for $\theta=26.25^{\circ}$.

tional truncated waveguide, which act as "magnetic mirrors" rors" of the metal disks in the present geometry. Similarly the electrically conducting walls of the cylindrical waveguide are replaced with a magnetic wall boundary condition on resonance. Due to the subwavelength thickness of the structure, the modes supported by the resonator presented here are TM polarized, with no requirement for quantization in the $z$-direction. They are analogous to the lowest energy TE modes of truncated waveguides. Indeed, to first order, the transverse fields and hence cutoff frequencies of the fundamental and higher harmonics may be calculated from the same waveguide theory. For instance, solving the wave equation for the $\mathrm{TM}_{n m 0}$ modes, once boundary conditions are imposed, yields a cutoff frequency for the fundamental mode of

$$
v_{\text {cutoff }}=\frac{p_{m n}^{\prime} c}{2 \pi a n},
$$

where $c$ is the speed of light, $a$ is the radius of the disk, and $n$ is the refractive index of the dielectric spacer. Here the factor $p_{m n}^{\prime}$ is the $m$ th root of an $n$th order Bessel function $J_{n}^{\prime}$, which must be solved numerically to define the cutoff frequencies pertaining to circular geometries. For the fundamental and second harmonic modes shown in Fig. 3 [parts (i) and (ii)], which we will label as the $\mathrm{TM}_{11}$ and the $\mathrm{TM}_{21}$, the values for $p_{m n}^{\prime}$ are 1.841 and 3.054, respectively. This first order approximation for the resonant frequencies of the modes supported describe all modes observed well (experimentally the resonant frequency of the fundamental mode occurs at $16.1 \mathrm{GHz}$, whereas Eq. (1) predicts a resonant frequency of $16.4 \mathrm{GHz}$ ) despite two important assumptions being made. The first is the approximation of the metal to a perfect conductor (in reality, there will be a finite penetration depth) and second is that the magnetic wall boundary conditions are also ideal. The latter is only truly valid if the limit of infinitesimal cavity thickness, otherwise fringing fields, result. $^{19}$

Parts (iii) and (iv) of Fig. 3 show the time averaged EM field magnitudes pertaining to modes $C(33.75 \mathrm{GHz})$ and $G$ $(63.2 \mathrm{GHz})$, respectively, calculated using the FEM model at $\theta=26.25^{\circ}, \phi=0^{\circ}$, with the incident plane wave being $p$-polarized. Recall that these two modes were absent from the $s$-polarized data shown in Figs. 1(b) and 2(b). From these field calculations it is clear that these modes are the $\mathrm{TM}_{01}$ and $\mathrm{TM}_{02}$, respectively, which resemble drum skin modes. Quantization in the radial direction implies a reversal in the direction of the electric and magnetic vectors over a distance equal to the radius $a$ in the case of the $\mathrm{TM}_{01}$ and $a / 2$ for the $\mathrm{TM}_{02}$ mode. Thus, in order to excite these modes, a corresponding phase variation in the incident electric vector (driving field) is required. In the $p$-polarized case this incident phase variation is achieved by means of a nonzero angle of incidence. However, in the $s$-polarized case, there is no such phase variation. Therefore modes $C$ and $G$ are excluded by symmetry.

To summarize, it has been shown that a metal-dielectric composite in the form of an array of metal disk resonators closely spaced by a dielectric from a ground plane can act as an efficient absorber of microwave radiation at selective wavelengths determined by the geometry of the disk. Unlike many available selective microwave absorbers, ${ }^{7}$ this structure is flexible, lightweight, and in our example, less than $1 / 130$ of the fundamental operating wavelength thickness (recall that at $\theta=26.25^{\circ}$, the fundamental resonant frequency occurs at $18.1 \mathrm{~mm}$ with the sample thickness being a mere $136 \mu \mathrm{m}$ in comparison). Highly localized modes, which have no quantization requirement with cavity height (z-direction), are shown to be supported at well defined wavelengths, with an EM response that is predominantly polarization, orientation, and incident angle independent.

M.J.L., A.P.H., and J.R.S. thank the Engineering and Physical Sciences Research Council, U.K. (EPSRC-GB) for their financial support.

${ }^{1}$ S. A. Long, M. W. McAllister, and L. C. Shen, IEEE Trans. Antennas Propag. 31, 406 (1983).

${ }^{2}$ M. W. Pospieszalski, IEEE Trans. Microwave Theory Tech. 27, 233 (1979).

${ }^{3}$ D. Rafizadeh, J. P. Zhang, R. C. Tiberio, and S. T. Ho, J. Lightwave Technol. 16, 1308 (1998)

${ }^{4}$ M. Rosenblit, Y. Japha, P. Horak, and R. Folman, Phys. Rev. A 73, 063805 (2006).

${ }^{5}$ R. K. Mongia, IEEE Antennas and Propagation-S/URSI Symposium Digest, Montreal, Canada, 1997 (unpublished), pp. 2202-2205.

${ }^{6}$ M. T. Lee, K. M. Luk, K. W. Leung, and M. K. Leung, IEEE Trans. Antennas Propag. 50, 1485 (2002).

${ }^{7}$ M. J. Lockyear, A. P. Hibbins, J. R. Sambles, and C. R. Lawrence, Appl. Phys. Lett. 83, 806 (2003).

${ }^{8}$ E. F. Knott, J. F. Schaeffer, and M. T. Tuley, in Radar Cross Section, edited by A. House, 2nd ed. (Artech House, Norwood, MA, 1993).

${ }^{9}$ Salisbury, U.S. Patent No. 2,599,944 (1952).

${ }^{10}$ A. P. Hibbins, J. R. Sambles, C. R. Lawrence, and J. R. Brown, Phys. Rev. Lett. 92, 143904 (2004).

${ }^{11}$ N. I. Landy, S. Sajuyigbe, J. J. Mock, D. R. Smith, and W. J. Padilla, Phys Rev. Lett. 100, 207402 (2008).

${ }^{12}$ J. Perini and L. S. Cohen, IEEE Trans. Electromagn. Compat. 35, 223 (1993).

${ }^{13}$ H. Komari and Y. Konishi, IEEE Trans. Broadcast. 40, 219 (1994).

${ }^{14}$ S. Pillai, K. R. Catchpole, T. Trupke, and M. A. Green, J. Appl. Phys. 101, 093105 (2007).

${ }^{15}$ www.ansoft.com.

${ }^{16}$ A. P. Hibbins, M. J. Lockyear, and J. R. Sambles, J. Appl. Phys. 99, 124903 (2006).

${ }^{17}$ D. M. Pozar, Microwave Engineering, 2nd ed. (Wiley, New York, 1998).

${ }^{18}$ D. Sievenpiper, L. Zhang, R. F. J. Broas, N. G. Alexopolous, and E. Yablonovitch, IEEE Trans. Microwave Theory Tech. 47, 2059 (1999).

${ }^{19}$ Y. Takakura, Phys. Rev. Lett. 86, 5601 (2001). 